\title{
Structure and Properties of Cellulose-Starch Blend Films Regenerated from Aqueous
}

\section{Sodium Hydroxide Solution}

\author{
Hitomi Miүamoto $^{1 *}$, Chihiro Yamane ${ }^{1}$, Masaharu Seguchi ${ }^{1}$ and Kunihiko OKaлima ${ }^{2}$ \\ ${ }^{1}$ Faculty of Home Economics, Kobe Women's University, Aoyama 2-1, Higashisuma Suma-ku, Kobe 654-8585, Japan \\ ${ }^{2}$ Faculty of Engineering, Tokushima Bunri University, Shido 1314-1, Sanuki-shi, Kagawa 100-0004, Japan
}

Received July 7, 2008; Accepted March 24, 2009

It was reported in 1984 that cellulose could be dissolved in aqueous sodium hydroxide solution when the intramolecular hydrogen bonds of cellulose were partially broken down by physical treatments such as steam explosion. Although there have been many studies on alkali-soluble cellulose, little is known about cellulose blends made from aqueous sodium hydroxide solution. Therefore, we investigated the structure and properties of cellulose-starch blend films regenerated from the solution. The blends had a porous structure with an average pore size increased from 1 to $6 \mu \mathrm{m}$ with increased starch content. These pores were observed separately from each other, leading to high water and oil absorbencies of these blend films (the oil absorbency of the blend film containing $50 \%$ starch was over $400 \%$, ten times that of cellulose film). The results of $X$-ray measurements, dynamic viscoelastic measurements, enzyme etching, and iodine staining suggested that cellulose and starch were miscible in the amorphous regions but incompatible in the crystalline regions of the cellulose-starch blend films.

Keywords: cellulose, starch, blend, edible cellulose, alkali solubility

\section{Introduction}

In recent years, there have been many efforts to find new, less-polluting cellulose solvents for producing fibers and films. In this context, an aqueous sodium hydroxide solution of specific concentration was first reported by Okajima and Kamide (1984) to be a cellulose solvent. Since then, numerous studies have been conducted on alkali-soluble cellulose, especially in aq. $\mathrm{NaOH}$ solution, and have yielded the following results: (1) degree of intramolecular hydrogen bonds in cellulose molecules controls the solubility of cellulose in aq. $\mathrm{NaOH}$ (Okajima et al., 1992); (2) steam explosion process effectively breaks these hydrogen bonds (Okajima et al., 1990); (3) cellulose dissolves in aq. $\mathrm{NaOH}$ (Kamide et al., 1987); (4) regenerated cellulose films from aq. $\mathrm{NaOH}$ have high crystallinity, highly developed intramolecular hydrogen bonds, and selective uniplanar orientation of (11̄0) planes of cellulose II crystal, and these structural parameters can be easily controlled by changing coagulation conditions such as coagulant concentration and temperature (Yang et al., 2007);

*To whom correspondence should be addressed.

E-mail: k4056003@suma.kobe-wu.ac.jp and (5) regenerated cellulose fiber from aq. $\mathrm{NaOH}$ solution has the mechanical properties comparable to commercially available regenerated cellulose fibers (Yamane et al., 1996a). From a technical viewpoint, regenerated cellulose fibers with good mechanical properties for textile use have been produced in a bench-scale pilot plant (Yamane et al., 1996b, 1996c).

The most advantageous aspect of the above-mentioned cellulose-dissolving system is the safety of $\mathrm{NaOH}$ both for the environment and for use in food processing. The other dissolving systems are not legally approved for food use since these systems employ certain chemicals such as carbon disulfide, cuprammonium hydroxide, N-methyl morpholine $\mathrm{N}$-oxide, and DMAc-lithium chloride which are not authorized to be used for food processing. A cellulose-corn starch blend named CEKICEL ${ }^{\text {TM }}$ (Hisano et al., 1991) was thus prepared as food materials from aq. $\mathrm{NaOH}$ solutions on a commercial scale for the first time. Certainly, there exist many cellulosic food additives (e.g., cellulose powder, microcrystalline cellulose, and cellulose derivatives), a small amount of which is added to food in order to improve its properties, that is they have never been used as food 
materials. Although CEKICEL ${ }^{\mathrm{TM}}$, a blend of cellulose and corn starch, is commercially used for imitation crab meat and Japanese horseradish, little is known about its structure and properties. Another probable advantageous aspect of the cellulose-corn starch blend films is that the films can be handled in hot water, while other edible polysaccharide films such as starch, pullulan, and agar films dissolve in hot water. Such hot water resistant films might offer a new field of food materials.

Although numerous attempts have been made for structural analysis of the blends between cellulose and synthetic polymer such as poly (vinyl alcohol) (Nishio et al., 1989a), poly (ethylene oxide) (Nishio et al., 1989b), and poly (acrylonitrile) (Nishio et al., 1986; Shin and Kondo, 1997), little is known about the structure of the cellulose-polysaccharide blends made from the aqueous $\mathrm{NaOH}$ solution system. The objective of this paper was to understand the structure of the cellulose-corn starch blend film prepared from cellulose/corn starch aq. $\mathrm{NaOH}$ solution with varied proportions of the polymers. In addition, the relationship between the structure and mechanical properties was also investigated, and a desirable structure for food materials was proposed.

\section{Materials and Methods}

Materials A softwood sulfite pulp (Nippon Paper Chemicals Co., Ltd.) was subjected to saturated steam treatment followed by steam explosion treatment. Steam explosion experiments were carried out as follows: $200 \mathrm{~g}$ (dry base) of the cellulose samples were put into a 1.2-L stainless digester vessel. Saturated water vapour at $200^{\circ} \mathrm{C}$ was then introduced into the vessel for $5 \mathrm{~min}$. After closing the vapour input valve, the ball valve connected to the discharging nozzle was abruptly opened and the treated cellulose was discharged, with the aid of pressure difference, into a 6-L cyclone receiver (Okajima et al., 1990). The apparatus was constructed by Japan Chemical Engineering \& Machinery Co., Ltd. (Japan). The steam-exploded pulp was refined by dilute aqueous sodium hydroxide solution in order to remove cellulose oligomers and hemi-cellulose to prepare alkalisoluble cellulose with $M_{\mathrm{v}}=5.2 \times 10^{4}$ (average degree of polymerization $=320$ ). Corn starch $($ starch, $85.2 \%$ (amylose, $22.6 \%$; amylopectin, $62.6 \%$ ); protein, $1.1 \%$; ash, $0.3 \%$; water, $13.4 \%$ ) was kindly supplied by J-Oil Mills, Inc.; the catalogue number was Y-3414s. Reagent grade sodium hydroxide, sulfuric acid, and the other chemicals were purchased from Kishida Chemical Co., Ltd. and were used without further purification.

Preparation of Cellulose-Starch Blend Films Cellulose aq. $\mathrm{NaOH}$ solution at the polymer concentration of $5.0 \%$ and the alkali concentration of $7.6 \%$ was prepared as follows: alkali-soluble cellulose was dispersed in cold $\left(-4^{\circ} \mathrm{C}\right)$, dilute (4\%) aq. $\mathrm{NaOH}$ solution. To the slurry thus obtained, precooled $\left(-18^{\circ} \mathrm{C}\right)$ and concentrated $(22 \%)$ aq. $\mathrm{NaOH}$ solution was added, and the mixture was stirred at $4500 \mathrm{rpm}$ by a high-speed mixer (T.K. Robomics, Primix Co., Ltd.) below $0^{\circ} \mathrm{C}$. Starch aq. $\mathrm{NaOH}$ solutions with the same composition $(5 \%$ polymer and $7.6 \% \mathrm{NaOH})$ as in the cellulose aq. $\mathrm{NaOH}$ solution were mixed into cellulose aq. $\mathrm{NaOH}$ solution so that the cellulose/starch ratio ranged from 100/0 to 50/50.

Each of the cellulose/starch aq. $\mathrm{NaOH}$ solutions was casted on a glass plate with a thickness of $500 \mu \mathrm{m}$. These were immersed gently in a coagulation bath containing $10 \%$ $\mathrm{H}_{2} \mathrm{SO}_{4}$ at $-5^{\circ} \mathrm{C}$ for $5 \mathrm{~min}$. Finally, the blend films were rinsed repeatedly with deionized water. The obtained fresh films were freeze-dried for water and oil absorbency and water permeability measurements. In addition, these films were press-dried for X-ray diffraction measurement and dynamic viscoelastic measurement. Press-dried films were obtained by placing fresh films between several filter papers and drying under compressed conditions of about $0.2 \mathrm{~kg} / \mathrm{cm}^{2}$ at $70^{\circ} \mathrm{C}$ for $30 \mathrm{~h}$.

Wide-Angle X-ray Diffraction (WAXD) Wide-Angle $\mathrm{X}$-ray Diffraction was measured by reflection and transmission methods.

Reflection method. Sample films were cut into particlelike sizes less than $c a$. $120 \mu \mathrm{m}$ so as to negate the influence from the crystalline orientation and recorded on an X-ray diffractometer (Rotaflex Ru-200PL, Rigaku Co., Ltd., Japan) equipped with scintillation counter under the following conditions: $\mathrm{CuK} \alpha$ (wave length $=0.154 \mathrm{~nm}$ ), $40 \mathrm{kV}, 24 \mathrm{~mA}$, and $2 \theta=8-28^{\circ}$. Crystallinity index was estimated by peak areas responsible for $(1 \overline{1} 0),(110),(020)$ planes, separated by the Lorents-Gaussian peak separation method.

Transmission method. Stacked ribbon $(0.5 \mathrm{~mm}$ wide $\times$ $10 \mathrm{~mm}$ long $\times 0.7 \mathrm{~mm}$ thick) of blend film was used for the measurement by the transmission method. X-ray diffraction patterns taken perpendicular and parallel (through and edge views, respectively) to the top surface of the films were recorded on an X-ray diffractometer (RAXIS IIC, Rigaku Co., Japan) equipped with an imaging plate under the following conditions: $\mathrm{CuK \alpha}, 40 \mathrm{kV}$, and $100 \mathrm{~mA}$.

Scanning Electron Microscopy (SEM) Scanning electron microscopic observations of the fracture surface of lyophilized films were carried out with a SEM (S-2250N, Hitachi High-Technologies Co., Japan). Samples were spattered with platinum and palladium in vacuo (10 nm thickness) before observation (E-102, Hitachi High-Technologies Co., Japan).

Tensile Strength Measurement The tensile strength of the fresh blend films, of square shape and $3 \times 20 \mathrm{~mm}$ in size, 
was measured using a tension meter (FGS-50V-L, Shimpo Co., Ltd.) under the following conditions: elongation speed, $20 \mathrm{~mm} / \mathrm{min}$; humidity, $60 \%$; temperature, $20^{\circ} \mathrm{C}$. The values of four measurements on each sample were averaged.

Water and Oil Absorbency The films were oven-dried and weighed $\left(W_{\mathrm{D}}\right)$, then immersed in distilled water or olive oil at $20^{\circ} \mathrm{C}$ for two days. Next, the films were centrifuged at $1000 \mathrm{G}$ for $10 \mathrm{~min}$ to remove excess water or oil, and reweighed $\left(W_{\mathrm{w}}\right)$. The water and oil absorbencies were estimated from equation (1).

$$
\text { Absorbency }(\%)=\left(W_{\mathrm{W}}-W_{\mathrm{D}}\right) / W_{\mathrm{D}} \times 100
$$

Dynamic Viscoelasticity Dynamic viscoelastic properties was evaluated by measuring temperature dependence of loss tangent $(\tan \delta)$ of the films by using a viscoelastic spectrometer (Model DVA-200, IT Keisoku Seigyo (Measurement-Control) Co. Ltd., Japan) under the following conditions: frequency (f), $10 \mathrm{~Hz}$; heating rate, $10^{\circ} \mathrm{C} / \mathrm{min}$; sample length, $20 \mathrm{~mm}$; sample width, $5 \mathrm{~mm}$; adding amplitude, 18 $\mu \mathrm{m}$; scanning temperature range, $-150 \sim 320^{\circ} \mathrm{C}$.

\section{Water Permeability}

The freeze-dried blend film as a filter was placed in a pressure filtration system (KST-47; max inlet, 0.49 MPa; max differential, $0.29 \mathrm{MPa}$; Advantech Co., Ltd., Japan) and the volume of water that passed through the filter was measured under the following conditions: pressure difference, $0.049 \mathrm{MPa}$; temperature, $25^{\circ} \mathrm{C}$. Commercial membrane filters were used for comparison: a reverse osmosis (RO) membrane (SU 800, Toray Industries, Inc., Japan), a nanofiltration (NF) membrane (NTR 7450, Nitto Denko Co., Japan), and a microfiltration (MF) membrane (Hipore ${ }^{\mathrm{TM}}$ 2000, Asahi Kasei Chemicals Co., Japan).

Enzymatic Etching of the Films In order to clarify how corn starch was dispersed in the blend films, starch was removed by enzymatic etching: the films were immersed in a 5\% $\alpha$-amylase (F11075V, Kishida Chemical Co., Ltd., Japan) solution and incubated at $40^{\circ} \mathrm{C}$ on a shaker $\left(90 \mathrm{~min}^{-1}\right)$ for 7 days.

\section{Results and Discussion}

Properties of the Cellulose-Starch Blend Films Almost all the starch remained in the blend films after their production, during which cellulose and starch were dissolved in aqueous sodium hydroxide and then coagulated by sulfuric acid followed by complete washing with water. Figure 1 shows the effect of starch content $\left(C_{\mathrm{st}}\right)$ on the loss in weight of the blend films from their initial values. Starch content expresses the initial weight ratio of starch to the total weight of cellulose and starch on dry base. The loss in weight of cellulose without starch $\left(C_{\mathrm{st}}=0 \%\right)$ was $2.4 \%$, probably due to the loss of the low molecular weight fraction of cellulose or hemi-cellulose. The weight loss of the films were 3.1, 4.2, $5.1,9.5$, and $9.2 \%$ for $C_{\mathrm{st}}=10,20,30,40$, and $50 \%$, respectively. This fact indicates that more than $80 \%$ of the original starch content was retained in the blend films. If cellulose and starch were not entangled, all the starch would be eluted from blend films, because starch alone cannot be coagulated by a $10 \% \mathrm{H}_{2} \mathrm{SO}_{4}$ coagulation solution. Therefore, it was suggested that, to some extent, cellulose and starch were entangled with each other in the blend films.

Figures $2 \mathrm{a}$ and $2 \mathrm{~b}$ show the water and oil absorbencies of

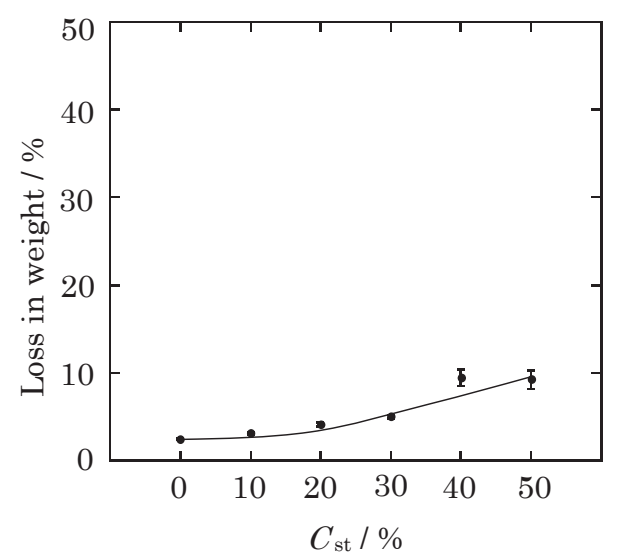

Fig. 1. Starch content $\left(C_{\mathrm{st}}\right)$ dependence of loss in weight of cellulose and cellulose-corn starch blend films during their production.

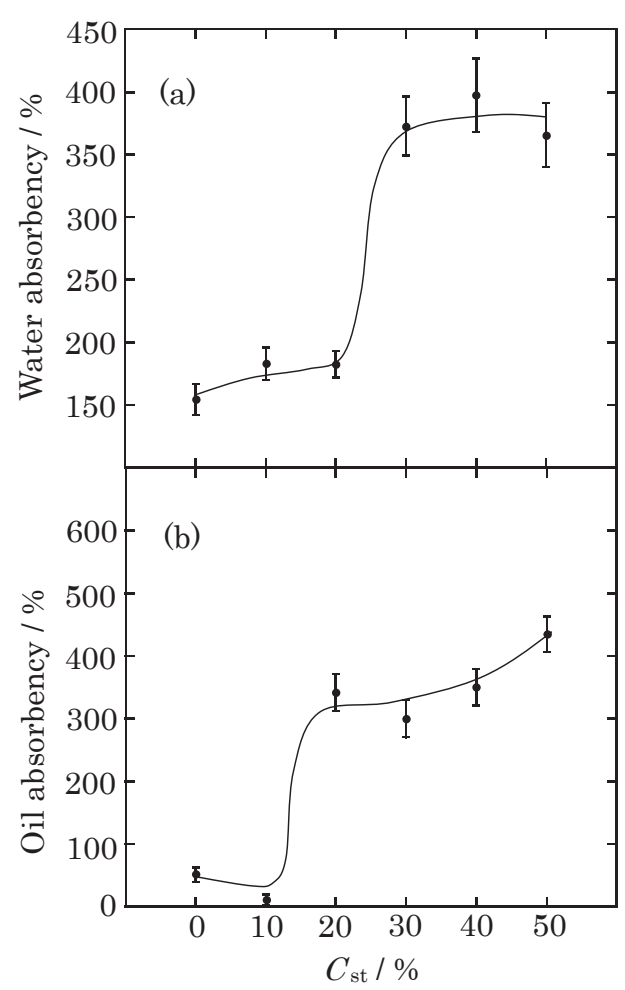

Fig. 2. $C_{\text {st }}$ dependence of water and oil absorbency of cellulose and cellulose-corn starch blend films: (a), water absorbency; (b), oil absorbency. 
the blend films, respectively. The water absorbency increased drastically at $C_{\mathrm{st}}=30 \%$ from 200 to more than $350 \%$. The blends with over $30 \%$ starch $\left(C_{\mathrm{st}} \geq 30 \%\right)$ swelled in water to about four times their original dry weight at $25^{\circ} \mathrm{C}$. The oil absorbency also rose sharply at $C_{\mathrm{st}}=20 \%$. Although the cellulose film $\left(C_{\mathrm{st}}=0 \%\right)$ absorbed oil only up to $40 \%$ of its dry weight, the absorbency of the blend film $\left(C_{\mathrm{st}}=50 \%\right)$ was over $400 \%$, i.e., 10 times more than that of the cellulose film $\left(C_{\mathrm{st}}=0 \%\right)$. In order to clarify the reason for these high absorbencies, the structures of these blends needed to be carefully taken into consideration. The above results led us to expect that the blends with a high starch content have a distinctive pore structure, as will be discussed later.

Comparing the blend films with commonly used food additives such as pulp, corn starch, and soy protein, it was found that the water absorbency of each additive was far lower than that of the blend at $C_{\mathrm{st}}=50 \%$. They were: $110 \%$ for pulp (fiber), 99\% for corn starch (powder), and 130\% for soy protein (powder). In general, water absorbency differs between sample shapes such as powder, fiber, and films; however, we confirm that the water absorbency of the cellulose-corn starch blend did not change despite the difference in shapes. This suggests that distinctively large pores in the blend structure at $C_{\mathrm{st}}=30-50 \%$, as will discussed later, are a primary factor of high water absorbency. These values were less than one-third of that of the blend film with $50 \%$ starch. That is, once cellulose and starch are assembled into a blend, a higher absorbency was achieved than could be attained by either component alone. When used as food additives, the blends may serve as absorbents for precooked or frozen foods, preventing liquid food ingredients from moving or the draining away of these ingredients during thawing.

Figures $3 \mathrm{a}$ and $3 \mathrm{~b}$ show the tensile strength (a) and elongation (b) of fresh blend films (no drying). The tensile strength decreased with increased $C_{\text {st }}$, particularly sharply at $C_{\text {st }}=30 \%$, the same $C_{\text {st }}$ at which water and oil absorbencies showed marked increases. Figures $3 \mathrm{c}$ and $3 \mathrm{~d}$ show the Young's modulus and breaking energy of the films. The density of these films was almost $1 \mathrm{~g} / \mathrm{cm}^{2}$, because these fresh films contained abundant water. The Young's modulus and breaking energy decreased with increased $C_{\mathrm{st}}$, and the latter greatly decreased around $C_{\mathrm{st}}=30 \%$. This indicated that a certain specific structural parameter of the blends would probably influence these properties. There were almost no changes in tensile elongation with differing starch content. However, the tensile strength and Young' modulus of the blend films $\left(C_{\mathrm{st}}\right.$ $=40$ and $50 \%$ ) were one-fifth of those of the cellulose film without starch $\left(C_{\mathrm{st}}=0 \%\right)$. Furthermore, the breaking energy of $C_{\mathrm{st}}=40$ and $50 \%$ were only one-hundredth of that of $C_{\mathrm{st}}$ $=0 \%$. When we ourselves chewed these low-strength films, they were swallowed quite easily and smoothly. In addition, the cellulose-corn starch blend films can be handled in hot water, while other edible polysaccharide films such as starch, pullulan, and agar films dissolve in hot water. Therefore, it is possible that cellulose-corn starch films could be used as new food materials.

Scanning Electron Microscopy (SEM) Fresh cellulosestarch blend films (including those without starch) were freeze-fractured and lyophilized. Figure $4 \mathrm{a}$ is the SEM micrograph of the freeze-fractured side surface of the cellulose

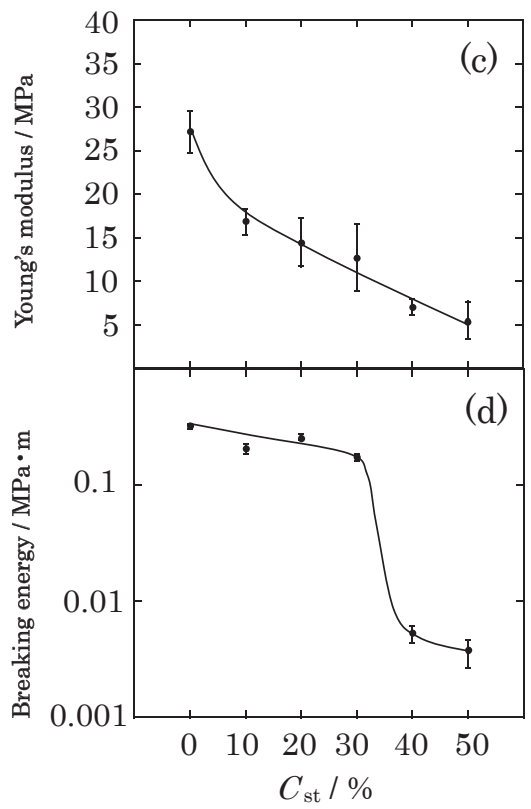

Fig. 3. $C_{\mathrm{st}}$ dependence of tensile strength (a), tensile elongation (b), Young's modulus (c), and breaking energy (d) of cellulose and cellulose-corn starch blend films. 


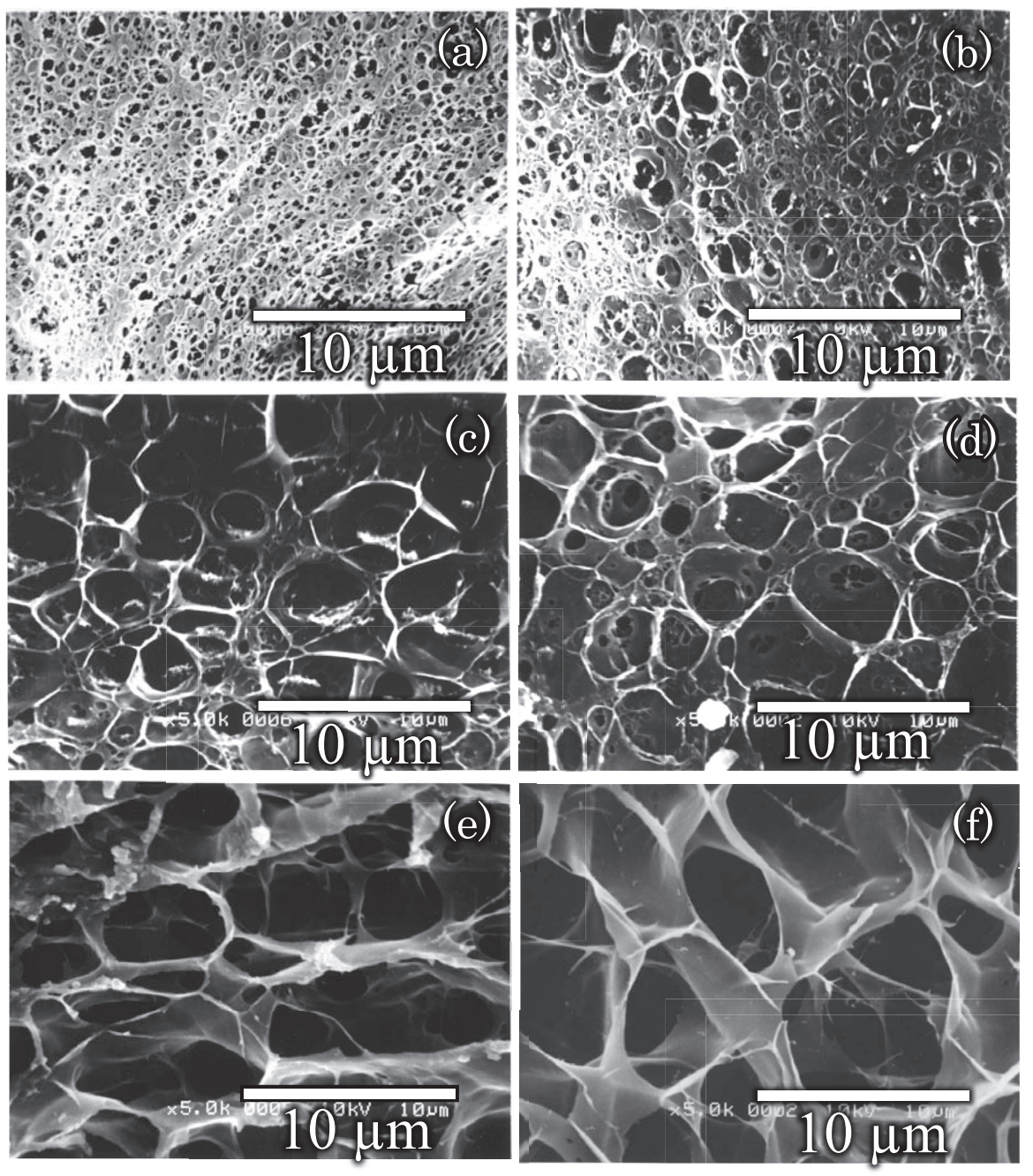

Fig. 4. SEM micrographs of side surfaces of cellulose and cellulose-corn starch blend films, freezefractured and lyophilized: (a), $C_{\mathrm{st}}=0 \%$; (b), $10 \%$; (c), $20 \%$; (d), $30 \%$; (e), $40 \%$; (f), $50 \%$.

film and Figures $4 \mathrm{~b}-4 \mathrm{f}$ are those of the blend films with $C_{\text {st }}$ ranging from 10 to $50 \%$. All films showed a complicated porous structure with pore sizes varying from ca. 0.01 to $15 \mu \mathrm{m}$. A closer look at these figures suggested that there appeared to be a certain difference between $C_{\mathrm{st}}=10 \%$ and $20 \%$. Specifically, round pores existed in the polymer matrices of the pure cellulose film (Figure 4a) and the $C_{\mathrm{st}}=10 \%$ film (Figure 4b), whereas the other films $\left(C_{\mathrm{st}}=20-50 \%\right)$ (Figure $4 \mathrm{c}-4 \mathrm{f}$ ) consisted of many pores (diameter $=1-15$ $\mu \mathrm{m}$, approximately) that were surrounded by thin membranes of ca. $0.2-1.0 \mu \mathrm{m}$ thickness. These porous structures were also observed by Transmission Electron Microscopy (TEM) analysis, in which the samples were encapsulated with blend resin and sliced into ultra-thin films of $60 \mathrm{~nm}$ thickness using a microtome. Figure 5 shows the effect of $C_{\mathrm{st}}$ on the average pore sizes of these films. The average pore size was calculated from 40 randomly selected pores in the SEM micrographs; the size of each pore is the average of

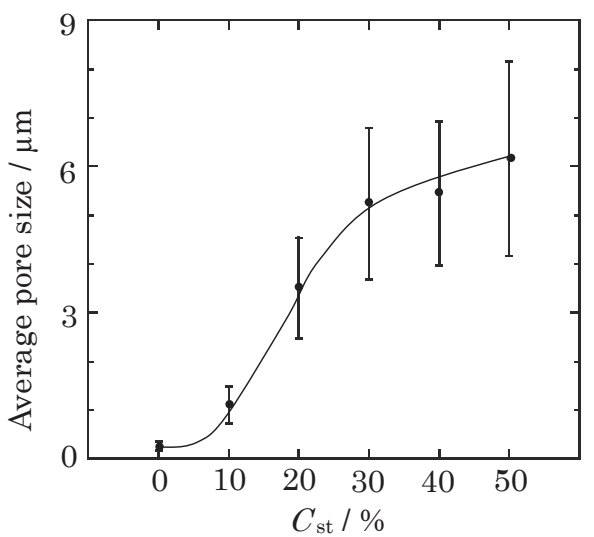

Fig. 5. $C_{\text {st }}$ dependence of average pore size of cellulose and cellulose-corn starch blend films.

the longest and shortest length of the pore. The average pore size increased markedly at $C_{\mathrm{st}}=20 \%$ and reached $6 \mu \mathrm{m}$ at $C_{\mathrm{st}}$ $=50 \%$. This $C_{\mathrm{st}}-$ dependency was quite similar to that ob- 
served for the water and oil absorbencies, indicating that the pore structure of these particular blends would be responsible for the high absorbencies. The structure and morphology of starch-free cellulose films and fibers, coagulated from cellulose aq. $\mathrm{NaOH}$ under various conditions, have been widely studied, revealing a rather denser structure than that of these cellulose-starch blend films. Specifically, the upper limit of the average pore size was less than $0.5 \mu \mathrm{m}$ (Yamashiki et al., 1992; Matsui et al., 1995). It is important to note that the cellulose-starch blend film was the only one among cellulosic materials to form such a macroporous structure.

Permeability Membranes for industrial use are classified according to pore size: microfiltration (MF) membrane, average pore size $>0.1 \mu \mathrm{m}$; ultrafiltration (UF) membrane, $2 \mathrm{~nm}-100 \mathrm{~nm}$; nanofiltration (NF) membrane, $0.5 \mathrm{~nm}-2$ $\mathrm{nm}$; and reverse osmosis (RO) membrane, $0.2 \mathrm{~nm}-0.5 \mathrm{~nm}$. In general, water permeability increases proportionally to increases in average pore size. In fact, the water permeability of regenerated cellulose films prepared by coagulation in acetone-water solution (pore size ca. $50 \mathrm{~nm}$ ) and sulfuric acid solution (pore size ca. $1 \mathrm{~nm}$ ) from a cellulose-cuprammonium solution were ca. 400 and $70 \mathrm{~mL} \cdot \mathrm{m}^{-2} \cdot \mathrm{hr}^{-1} \cdot \mathrm{hPa}^{-1}$, respectively. These values were in the range of UF and NF. According to the above-mentioned classification, the cellulose and cellulose-starch blend films in this study would be categorized as MF membranes since the pore size was more than $0.1 \mu \mathrm{m}$. Although they were expected to have high water permeability (comparable to that of MF) because of their large pore sizes, the permeability of the blend with $50 \%$ starch was comparable to that of the RO membrane as shown in Table 1. This indicated that these pores were isolated from each other and separated by the thin membrane as observed in Figure 4(f). It is reasonable to conclude that the higher water and oil absorbencies of the blend films than those of pure cellulose film, as shown in Figure 4(a), could be attributed to their isolated macropores, which prevented the liquids from draining off. The mechanism responsible for the formation of such porous structures was not readily identifiable. However, the mechanism could be explained using either thermodynamics or molecular dynamics.

Table 1. Water permeability of cellulose-corn starch blend film and other commercially available films.

\begin{tabular}{cc}
\hline Films & $\begin{array}{c}\text { Water permeability / } \\
\mathrm{ml} \cdot \mathrm{m}^{-2} \cdot \mathrm{hr}^{-1} \cdot \mathrm{hPa}^{-1}\end{array}$ \\
\hline Cellulose-corn starch blend film & 1 \\
$C_{\text {st }}, 50 \%$ & 0.5 \\
RO membrane & 11 \\
NF membrane & 2500 \\
MF membrane & \\
\hline
\end{tabular}

The first mechanism is based on the micro-phase separation theory (Kamide, 1990). In general, a polymer solution is divided into a phase with a high polymer concentration (polymer-rich phase) and a phase with a low polymer concentration (polymer-lean phase) due to phase separation. Kamide reported that there were two kinds of structural formation processes due to micro-phase separation depending on the initial polymer concentration of the solution. That is, either a polymer-rich phase or a polymer-lean phase will initially appear as a dispersed phase from the solution. When the initial polymer volume fraction is larger than at the specific polymer fraction called the critical solution point, the polymer-lean phase appears. Since this phase has a comparatively low viscosity than the original polymer solution, the particles of the polymer-lean phase rapidly become larger by amalgamation. These aggregates are themselves spherical pores surrounded by polymer-rich membranes. This mechanism can be expected to produce large, isolated pores, leading to lower water permeability despite the porous structure. Therefore, this mechanism would seem to apply to these cellulose-starch blends.

In the second mechanism, molecular sheet structure, which has been identified by Hayashi et al., (1974) and Hermans (1949) as the basic feature (called plane lattice or ribbon-shaped structures) of regenerated cellulose, may play an important role in the formation of the porous structures. As the coagulation proceeds, several molecular sheets stack progressively to form thin membranes, and these membranes then randomly disperse in solution and stick together upon contact to form the three-dimensional network (Kiyose et al., 2007). This process has been referred to as diffusion-limited cluster-cluster aggregation of particles (Kolb et al., 1983). Thus, the formation of porous structures of the blend films may be explained from two angles.

Wide-Angle X-ray Diffraction (WAXD) Figure 6 shows the WAXD patterns of cellulose and cellulose-corn starch blends cut into particle-like sizes in order to negate the influence of crystalline orientation as measured by the reflection method. The WAXD patterns, except that of corn starch, showed cellulose II polymorph defined by three major dif-

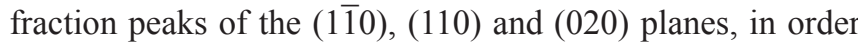
of increased diffraction angle. It is noteworthy that the crystal form of regenerated cellulose, prepared from cellulose solution such as this dissolving system, is cellulose II polymorph. Cellulose II crystal has two antiparallel chains organized in a $P 2_{1}$ space group and unit cell parameters: $\mathrm{a}=0.801$ $\mathrm{nm}, \mathrm{b}=0.904 \mathrm{~nm}, \mathrm{c}=1.036 \mathrm{~nm}$, and $\gamma=117.1^{\circ}$ (Langan et al., 1999). The diffraction pattern of cellulose remained unchanged even when corn starch was blended into cellulose, suggesting that cellulose and corn starch were not compatible 


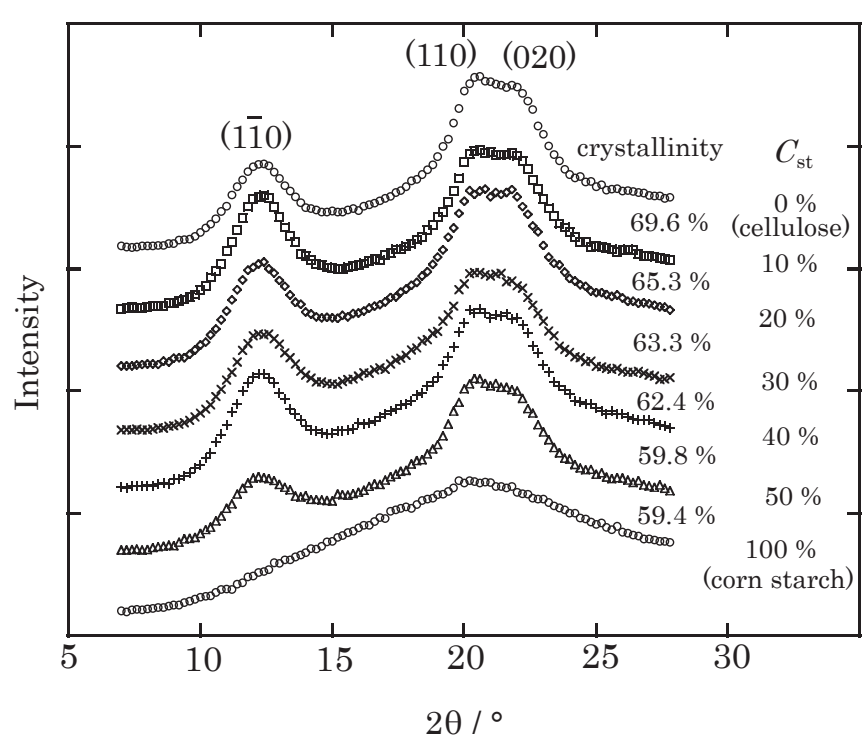

Fig. 6. WAXD patterns of cellulose and cellulose-corn starch blends cut into particle-like sizes in order to negate the influence of crystalline orientation and measured by the reflection method.

in the crystalline regions. The one broad peak of corn starch indicated its complete amorphousness.

Figures $7 \mathrm{a}-7 \mathrm{~d}$ are edge-view wide angle $\mathrm{X}$-ray diffraction (EV-WAXD) and through-view wide angle X-ray diffraction (TV-WAXD) patterns of both cellulose and cellulose-starch blends. The films were placed in parallel to the beam stopper for the EV-WAXD measurements. In the EV-WAXD patterns, the (110) arc on the equator and the (110) arc on the meridian were observed. On the other hand, the TV-WAXD pattern was missing the (1 $1 \overline{1} 0)$ reflection, whereas rather strong (110) and (020) doublet rings were visible. Thus, it could be concluded that the (110) planes of cellulose II crystals both in the cellulose and cellulose-starch blends were preferentially oriented parallel to the film surface. In other words, (110) plane was oriented perpendicular to the film, because (110) and (110) planes intersect almost vertically. According to earlier papers (Yamane et al., 2006; Kiyose et al., 2007), the orientation of the crystal planes takes place through the next three steps: (i), formation of cellulose molecular sheets; (ii), stacking of the molecular sheets to form planar crystal; and (iii), shrinkage of coagulating films perpendicular to the film surface brings planar orientation of the crystal planes. Although the diffraction intensity from the blend was slightly lower than that from the cellulose film, both types of films had similar diffraction profiles with each other. Thus, starch had little effect on the cellulose crystal region. This would indicate that there was no compatibility of the crystalline regions of the blends; if compatibility exists, intermolecular interaction between cellulose and (a)

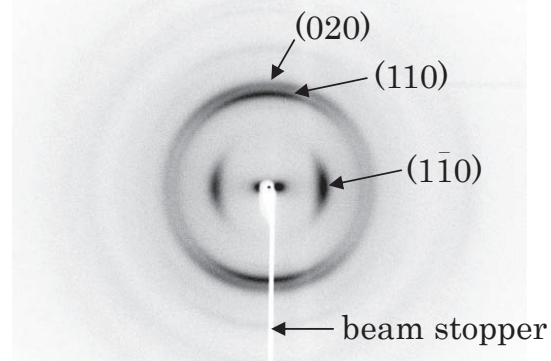

(c) (b)

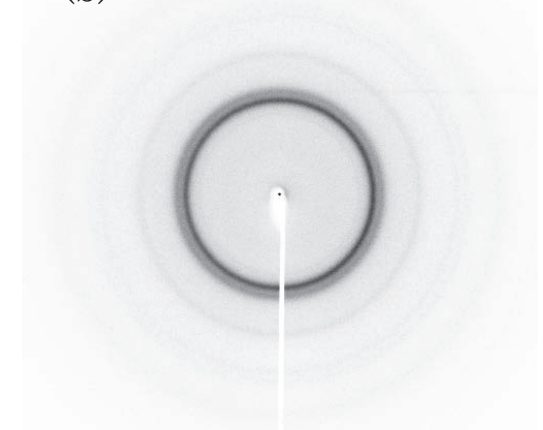

(d)

Fig. 7. WAXD patterns of cellulose and cellulose-corn starch blend films measured by a transmission method. The films were irradiated parallel and perpendicular (Edge View (EV) and Through View (TV), respectively) to the top surface of the films: (a), $C_{\mathrm{st}}=0 \%, \mathrm{EV}$; (b), $C_{\mathrm{st}}=0 \%, \mathrm{TV} ;(\mathrm{c}), C_{\mathrm{st}}=50 \%, \mathrm{EV}$; (d), $C_{\mathrm{st}}=50 \%, \mathrm{TV}$. 
starch would occur in amorphous regions. The amorphous regions of regenerated cellulose were reported to be divided into four segmental domains, where the density of intra- and intermolecular hydrogen bonds was different from each other (Manabe et al., 1986). However, these amorphous regions in cellulose-corn starch blend films cannot be separated quantitatively, and corn starch is almost amorphous, as shown in the WAXD patterns in Figure 6. Therefore, the regions in the blend films, other than cellulose crystal, were defined as amorphous regions in the present study.

Dynamic Viscoelasticity Figures $8 \mathrm{a}-8 \mathrm{c}$ show the temperature dependence of loss tangent $(\tan \delta)$ in the dynamic viscoelasticity measurement for the cellulose film (7a), starch film (7b), and cellulose-starch blend film $\left(C_{\mathrm{st}}=50 \%, 7 \mathrm{c}\right)$, respectively. There were observed three dynamic absorption peaks and shoulder for the cellulose film tentatively named $\alpha_{\text {cell }}, \alpha_{\text {sh }}$, and $\beta$ in reverse order of temperature. These absorptions were assigned by Manabe et al., (1986) as follows: $\alpha_{\text {cell }}$, Micro-Brownian motion of cellulose chain in amorphous region with highly developed intra- and intermolecular hydrogen bonds; $\alpha_{\mathrm{sh}}$, Micro-Brownian motion of cellulose chain in amorphous region with moderately developed intra- and intermolecular hydrogen bonds; and $\beta$, local twisting motion of cellulose chain. For the starch film, each absorption peak tentatively named $\alpha_{\mathrm{st} 1}, \alpha_{\mathrm{st} 2}$ or $\beta$ was observed in reverse order of temperature. The assignments of these absorptions have not yet been completed. However, the absorption peaks of the cellulose film shifted to a lower temperature and those of the starch film moved to a higher temperature when cellulose and starch were blended into the film. These results indicate

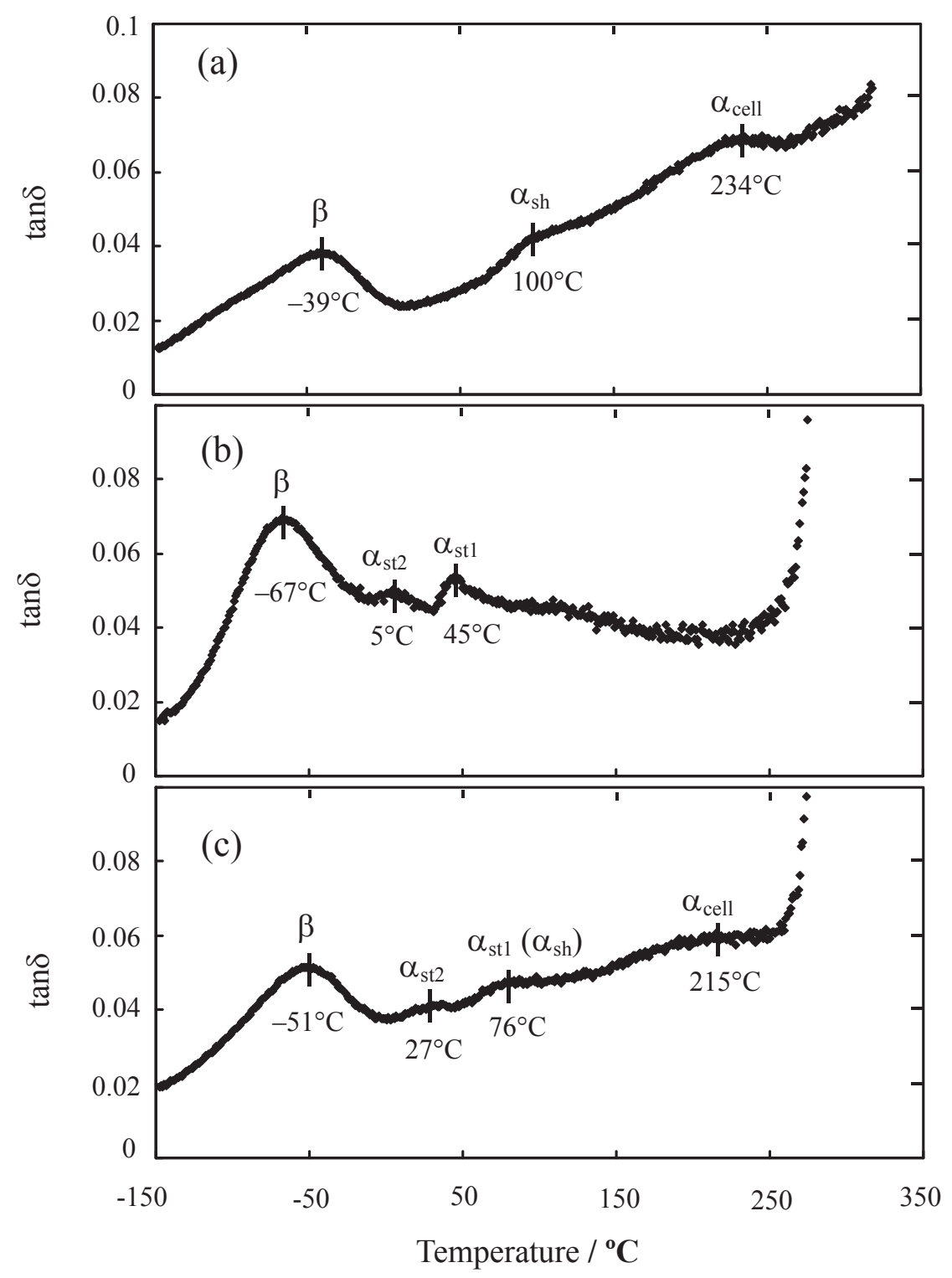

Fig. 8. Temperature dependence of loss tangent $(\tan \delta)$ for the sample films: (a), cellulose film; (b), the starch film; (c), the cellulose-starch blend film $\left(C_{\mathrm{st}}=50 \%\right)$. 
that the blend exhibited certain miscibility, which was presumed to be due to the capability of the two polymers, each with abundant hydroxyl groups, to interact mutually through hydrogen bonding in the amorphous regions. In order to clarify the blended state of the films, differential scanning calorimetry (DSC) measurement was carried out. However, no endothermic transitions attributed to gelatinization of starch was observed by DSC measurement. This might result from the pregelatinization of starch by the alkali dissolution or strong interaction between cellulose and starch in the blend. In general, thermal transition peaks by DSC measurement can not be detected for cellulosic materials.

Enzymatic Etching of the Films In order to clarify the state of mixing between cellulose and starch in the blend film, the starch was removed from the film using amylase treatment. The weight decreased by $23.4 \%$ for $C_{\mathrm{st}}=30 \%$, $42.1 \%$ for $C_{\mathrm{st}}=50 \%, 68.2 \%$ for $C_{\mathrm{st}}=70 \%$ and $100 \%$ for $C_{\mathrm{st}}$ $=100 \%$ (i.e., the starch film disintegrated completely). These decreases in weight were approximately equivalent to the respective starch content of the blend films. However, a closer look at this result revealed that the differences between decreases in weight and starch content were slightly larger for the smaller values of $C_{\mathrm{st}}$. This implies that certain molecular interactions may have existed between cellulose and starch molecules, inhibiting the degradation or draining out of the starch. Figures $9 a-9 d$ are SEM micrographs of the freeze- fractured and lyophilized side surface of the cellulose-starch blend with $C_{\mathrm{st}}=50 \%$, before (Figure 9a, b) and after (Figure $9 \mathrm{c}, \mathrm{d})$ amylase treatment. The morphological features of the film, before and after enzyme etching, showed no significant differences, suggesting that it existed in a homogeneous and finely dispersed form in the cellulose matrix. The distribution of starch was also examined in a $C_{\mathrm{st}}=50 \%$ film stained with iodine using an SEM equipped with an extended period energy dispersive X-ray (EDX) analysis system. The analysis of iodine in the blend film showed that iodine (which binds to the starch fraction) existed homogeneously at submicrometer resolution.

\section{Conclusions}

Cellulose and cellulose-corn starch blend films were prepared from aqueous sodium hydroxide solution followed by coagulation with sulfuric acid. The structure and properties of these films were studied, mainly using SEM, WAXD, and dynamic viscoelastic measurement. The following results were obtained:

1) Cellulose-starch blend films had a porous structure with an average pore size increased from 1 to $6 \mu \mathrm{m}$ with increased starch content.

2) These pores existed separately from each other, which could contribute to the high water and oil absorbencies of the blend films (the oil absorbency of the $C_{\mathrm{st}}=50 \%$ blend film
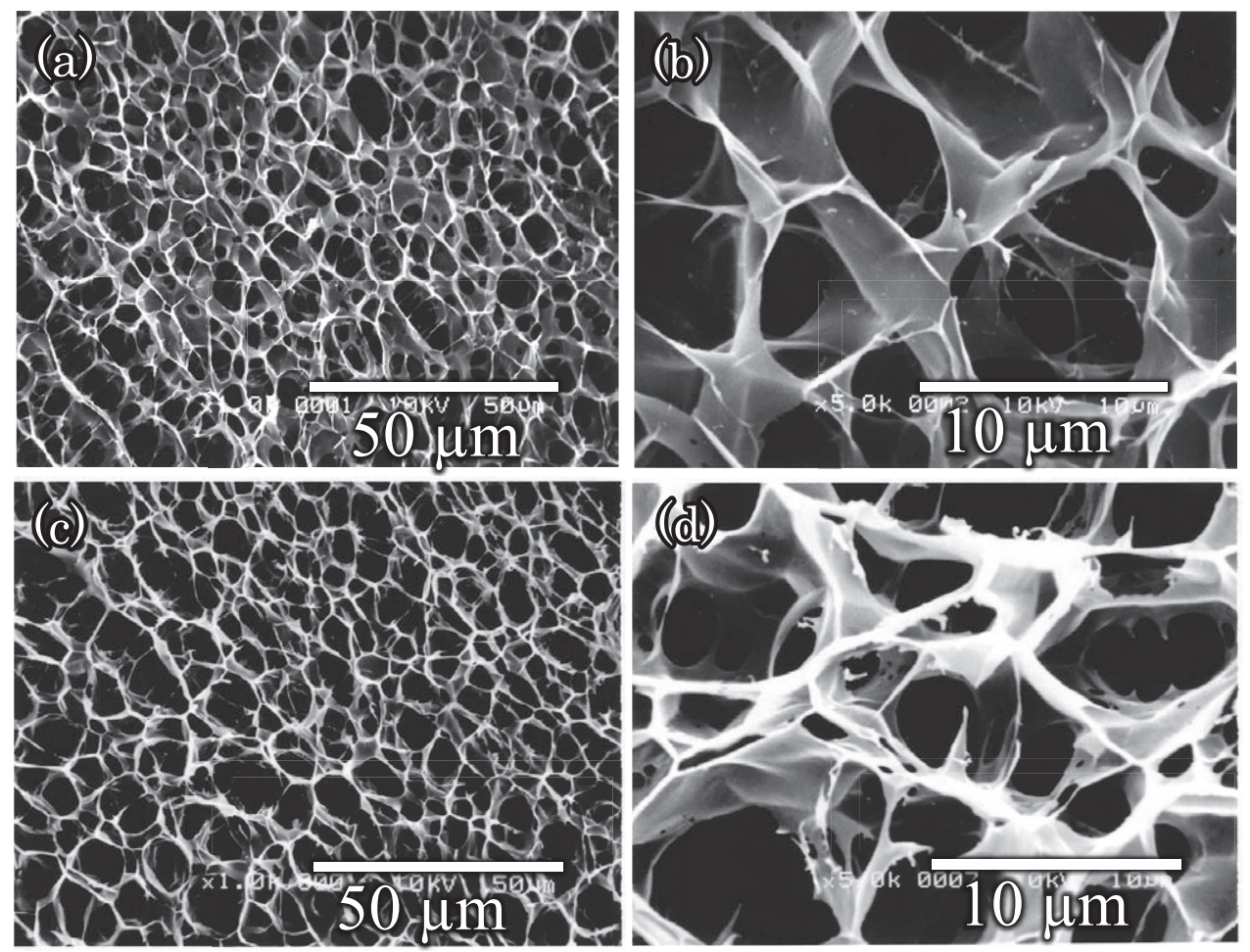

Fig. 9. SEM micrographs of the freeze-fractured and lyophilized side surface of a cellulose-corn starch blend film with $C_{\mathrm{st}}=50 \%$, before (a, b) and after (c, d) $\alpha$-amylase treatment. 
was over $400 \%$, ten times higher than that of $C_{\text {st }}=0 \%$, i.e., cellulose film).

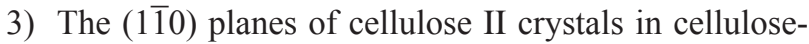
starch blend films were preferentially oriented parallel to the film surface. The degree of uniplanar orientation and crystallinity of these blends were almost the same as those of cellulose films, indicating that there was no compatibility of the crystalline regions of the blends.

4) The shifts in temperature of the absorption peaks of $\tan \delta$ measured by dynamic viscoelastic measurements, and the uniform and homogenous appearances of enzyme etched and iodine stained films observed by SEM revealed that cellulose and starch were miscible to a certain extent in the amorphous region.

In summary, cellulose-polysaccharide blends are expected to be of wide use as new food materials. The structure and properties of the blends and related materials are technologically and scientifically important.

\section{References}

Hayashi, J., Masuda, S., and Watanabe, S. (1974). Plane lattice structure in amorphous region of cellulose fiber. Nippon Kagaku Kaishi, 5, 948-954.

Hermans, P.H. (1949). Degree of lateral order in various rayons as deduced from X-ray measurements. J. Polym. Sci., 4, 145-151.

Hisano, J., Goto, A. and Okajima, K. (1991). Edible body and process for preparation thereof, United States Patent 4994285.

Jong-Ho, Shin., and Kondo, T. (1997). Cellulosic blends with poly(acrylonitrile): characterization of hydrogen bonds using regioselectively methylated cellulose derivatives. Polym., 39 (11), 6899-6904.

Kamide, K., Saito, M. and Kowsaka, K. (1987). Temperature dependence of limiting viscosity number and radius of gyration for cellulose dissolved in aqueous $8 \%$ sodium hydroxide solution. Polym. J., 19 (10), 1173-1181.

Kamide, K. (1990). Thermodynamics of polymer solution, phase equilibria and critical phenomena. (pp. 450-454). Tokyo: Elsevier.

Kiyose, M., Yamamoto, E., Yamane, C., Midorikawa, T. and Takahashi, T. (2007). Structure and properties of low-substituted hydroxypropylcellulose films and fibers regenerated from aqueous sodium hydroxide solution. Polym. J., 39 (7), 703-711.

Kolb, M., Botet, R. and Jullian, R. (1983). Scaling of kinetically growing clusters. Physical Review Letters, 51 (13), 1123-1126.

Langan, P., Nishiyama, Y. and Chanzy, H. (1999). A revised structure and hydrogen-bonding system in cellulose II from a neutron fiber diffraction analysis. J. Am. Chem. Soc., 121, 9940-9946.
Manabe, S., Iwate, M. and Kamide, K. (1986). Dynamic mechanical absorption for regenerated cellulose solids in the temperature range from 280 to $600 \mathrm{~K}$. Polym. J., 18 (1), 1-14.

Matsui, T., Sano, T., Yamane, C., Kamide, K. and Okajima, K. (1995). Structure and morphology of cellulose films coagulated from novel cellulose/aqueous sodium hydroxide solution by using aqueous sulfuric acid with various concentrations. Polym. J., 27 (8), 797-812.

Nishio, Y., Roy, S.K. and St John Manley, R. (1986). Blends of cellulose with polyacrylonitrile prepared from $N, N$-dimethylacetamide-lithium chloride solutions. Polym., 28 (7), 1385-1390.

Nishio, Y., Haratani, T. and Takahashi, T. (1989a). Cellulose/ poly(vinyl alcohol) blends: an estimation of thermodynamic polymer-polymer interaction by melting point depression analysis. Macromolecules, 22 (5), 2547-2549.

Nishio, Y., Hirose, N. and Takahashi, T. (1989b). Thermal analysis of cellulose/poly(ethylene oxide) blends. Polym. J., 21 (4), 347-351.

Okajima, K. and Kamide, K. (1984). Cellulose dope, process for preparation and method for application thereof. United States Patent, 4634470.

Okajima, K., Yamashiki, T., Matsui, T., Saitho, M. and Kamide, K. (1990). Characterization of cellulose treated by the steam explosion method. Br. Polym. J., 22, 73-83.

Okajima, K., Kamide, K. and Kowsaka, K. (1992). Dissolution of natural cellulose into aqueous alkali solution: role of supermolecular structure of cellulose. Polym. J., 24 (1), 71-86.

Yamane, C., Mori, M., Saito, M. and Okajima, K. (1996a). Structure and mechanical properties of cellulose filament spun from cellulose/aqueous $\mathrm{NaOH}$ solution system. Polym. J., 28 (12), 1039-1047.

Yamane, C., Saito, M. and Okajima, K. (1996b). Industrial preparation method of cellulose - alkali dope with high solubility. Sen'i Gakkaishi, 52 (6), 310-317.

Yamane, C., Saito, M. and Okajima, K. (1996c). New spinning process of cellulose filament production from alkali soluble cellulose dope-net process. Sen'i Gakkaishi, 52 (7), 378-384.

Yamane, C., Aoyagi, T., Ago, M., Sato, K., Okajima, K. and Takahashi T. (2006). Two different surface properties of regenerated cellulose due to structural anisotropy. Polym. J., 38 (8), 819-826.

Yamashiki, T., Matsui, T., Kowsaka, K., Saitoh, M., Okajima, K. and Kamide, K. (1992). New class of cellulose fiber spun from the novel solution of cellulose by wet spinning method. J. Appl. Polym. Sci., 44, 691-698.

Yang, G., Miyamoto, H., Yamane, C. and Okajima, K. (2007). Structural changes of cellulose from aq. $\mathrm{NaOH}$ solution system. Polym. J., 39 (1), 34-40. 